\title{
Measurements and Estimation of the Extremely Low Frequency Magnetic Field of the Overhead Power Lines
}

\author{
G. Petrović ${ }^{1}$, T. Kilić ${ }^{1}$, T. Garma ${ }^{1}$ \\ ${ }^{I}$ Department of Power Engineering, FESB, University of Split, \\ R. Boskovica 32, 21000 Split, Croatia \\ tkilic@fesb.hr
}

\begin{abstract}
Paper reports the simple model to estimate the value of the Extremely low frequency magnetic field of the overhead power line. The model is based on the application of the Biot-Savart law, considering the actual pillar head geometry and the conductors mutual position as well as their catenary shape. Additionally, it is shown that influence of the catenary on the location of the sag maxima can be neglected. The accuracy of the proposed model is verified by measuring the magnetic field of $110 \mathrm{kV}$ and $400 \mathrm{kV}$ overhead power line. Moreover, statistical analysis of the overhead power line current has been performed in order to determine accurate current magnitude and consecutive radiation dose. The obtained results are compared to limits directed by corresponding Croatian laws.
\end{abstract}

Index Terms-Extremely low frequency, electromagnetic (EM) field, overhead power line model, Biot-Savart law, magnetic field measurements.

\section{INTRODUCTION}

Every active power object is a source of the electromagnetic (EM) field. While on high frequencies EM field represents compact entity, on the extremely low frequencies (ELF) one may treat electric and magnetic field as separate physical quantities [1]. The influence of the EM fields to living organisms significantly depends on frequency. During past few decades, researcher's attention has been focused on the magnetic field biological effects [2], [3]. Generally speaking, the magnetic fields are considered to present more significant risk for living beings compared to the corresponding electric fields [4]. International Agency for Research in Cancer (IARC) report [5] made World Health Organization to classify magnetic fields into $2 \mathrm{~B}$ category, meaning potentially carcinogenic.

$\mathrm{Up}$ to date, most of the European countries accepted recommendation of the International Commission for Nonionizing Radiation Protection (ICNIRP), which regulates allowed values of the magnetic field. According to available data [6], certain types of domestic appliances (hair-driers, shaving machines, etc.) may induce fields of the magnitudes

Manuscript received December 30, 2012; accepted April 15, 2013.

This research was funded by Croatian Ministry of Science, Education and Sport, project Modeling and Environmental Aspects of the ELF Magnetic Fields (No. 023-0231582-2294). significantly above the limits. However, these exposures are of short duration. Considering all electric power facilities, overhead power lines present the permanent and the most important influence on the general population.

The available literature reports three concepts for modelling overhead power line EM field. The first one consists of static and quasi-static models considering the electric and magnetic field separately [7], and approximating the conductors as the straight lines, i.e. neglecting sag. The alternative method, so called Transmission Line Method (TLM), assumes the conductors much longer than their mutual distance, uses the distributed parameters and treats the EM field by neglecting radiation [8], [9]. Finally, third method uses the antenna model (thinwire model) which enables time and frequency-domain calculations. In this case, strict EM field analysis is mandatory [10].

This paper reports the model of two most commonly used types of pillars, fir and Y, considering the actual geometry of the pillar head and conductor sag. All obtained results for the magnetic induction are compared to both measurements as well as limits directed by corresponding Croatian regulations [11].

\section{OVERHEAD POWER LINE MODEL}

Calculation of the magnetic induction generated by current flowing through 2 straight parallel conductors is based on expression [14]

$$
\begin{aligned}
& \mathbf{B}=-\frac{\mu_{o}}{2 \pi} \sum_{n=1}^{N} I_{n} \cdot\left\{\left[\frac{\left(y-d_{n}\right)}{r_{c n}^{2}}-\frac{\left(y+d_{n}+\alpha\right)}{r_{i n}^{2}}\right] \mathbf{i}-\right. \\
& \left.-\left[\frac{\left(x-h_{n}\right)}{r_{c n}^{2}}-\frac{\left(x-h_{n}\right)}{r_{i n}^{2}}\right] \mathbf{j}\right\},
\end{aligned}
$$

where $\mu_{0}$ and $I_{n}$ stand for the air permeability and each conductor current, respectively.

We will assume conductors perpendicular to $\mathrm{XY}$ Cartesian plane and define $\mathbf{i}$ and $\mathbf{j}$ as the corresponding unit vectors. The coordinates of the conductor element are $\left(h_{n}\right.$, $\left.d_{n}\right)$. Distance from the referent point $(\mathrm{x}, \mathrm{y})$ where the field is considered to the conductor element i.e. its image in the conductive half-space is given by : 


$$
\left\{\begin{array}{l}
r_{c n}=\sqrt{\left(x-h_{n}\right)^{2}+\left(y-d_{n}\right)^{2}}, \\
r_{i n}=\sqrt{\left(x-h_{n}\right)^{2}+\left(y+d_{n}+\alpha\right)^{2}} \\
\alpha=503 \sqrt{\frac{2 \rho_{g}}{f} \exp \left(-j \frac{\pi}{4}\right)},
\end{array}\right.
$$

where $\rho_{g}$ and $f$ stand for ground resistivity and current frequency, respectively.

Considering typical values of the ground resistances (10 $\Omega \mathrm{m}-5000 \Omega \mathrm{m})$, when calculating magnetic induction in the vicinity of the overhead power line, influence of the imaging may be neglected [12].

Besides current flowing through the conductors and pillar head geometry, the field calculation should include influence of the conductor sag, path direction modifications and the possible overhead power lines route intersections. Due to this, to obtain the reliable magnetic induction calculation, technique based on discrete approximation of the Biot-Savart law is chosen. Fig. 1 depicts 1 segment of the streamline as the power line conductor element in Cartesian coordinate system.

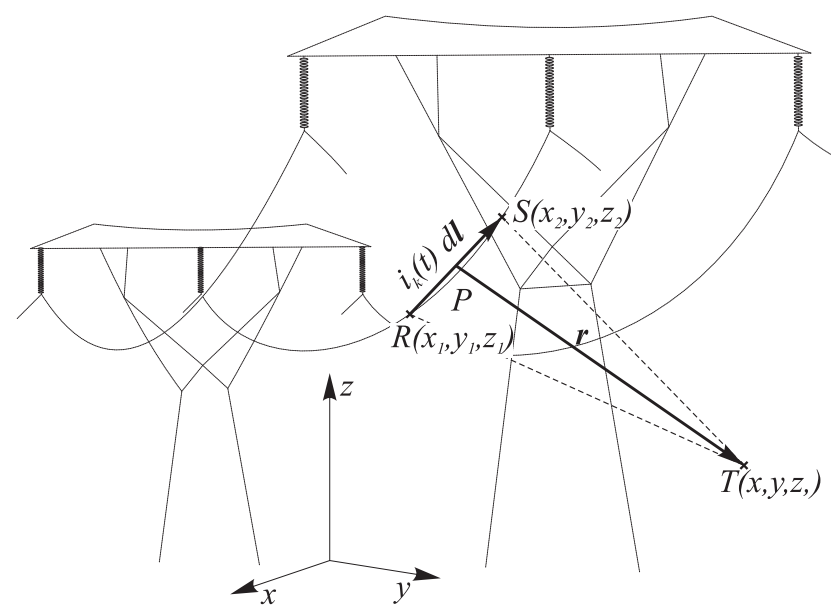

Fig. 1. Segment of the streamline as power line conductor segment.

According to Biot-Savart law, one segment of the plane streamline with the sufficiently short length generates following magnetic induction in arbitrary point of space

$$
d \mathbf{B}=\frac{\mu_{0} I}{4 \pi} \frac{d \mathbf{l} \times \mathbf{r}}{|\mathbf{r}|^{3}},
$$

where $d \mathbf{l}$ and $\mathbf{r}$ stand for the orientated streamline element and its distance to the point of view, respectively.

To circumvent the issue of conductor shape in the gravitational field (catenary equation [13]), we apply following reasoning: considering time-dependent current, solution of equation (3) in Cartesian system may be described by

$$
b_{k}(t)=\frac{\mu_{0} i_{k}(t)}{4 \pi T P}\left(\frac{R P}{R T}+\frac{P S}{S T}\right)
$$

where $T P, R P, R T, P S$ and $S T$ represent the distances between points according to Fig. 1. These distances are obtained from vectors RS, RT and ST whose initial and final point coordinates are given as input data:

$$
\left\{\begin{array}{l}
\mathbf{R S}=\left(x_{2}-x_{1}\right) \mathbf{i}+\left(y_{2}-y_{1}\right) \mathbf{j}+\left(z_{2}-z_{1}\right) \mathbf{k}, \\
\mathbf{R T}=\left(x-x_{1}\right) \mathbf{i}+\left(y-y_{1}\right) \mathbf{j}+\left(z-z_{1}\right) \mathbf{k}, \\
\mathbf{S T}=\left(x-x_{2}\right) \mathbf{i}+\left(y-y_{2}\right) \mathbf{j}+\left(z-z_{2}\right) \mathbf{k},
\end{array}\right.
$$

where $\boldsymbol{i}, \boldsymbol{j}, \boldsymbol{k}$ stand for the unit vectors in the direction of the Cartesian axes. Distance from the points $R P$ can be determined from the projection $\mathbf{R T}$ on vector $\mathbf{R S}$, meaning from scalar product

$$
R P=\frac{\mathbf{R T} \cdot \mathbf{R S}}{R S}
$$

Distance $P S$ is than given by: $P S=R S-R P$, while distance $T P$ is given by

$$
T P=\sqrt{R T^{2}-R P^{2}} .
$$

This enables the determination of the magnetic induction on the given location. However, in order to superimpose contributions from all segments, it is mandatory to determine both orientation and the value of the vector. The orientation of the magnetic induction vector is perpendicular to plane defined by vectors RS and ST, meaning it is equal to orientation of their vector product. Hence, it is necessary to determine cosines of the angles formed by aforementioned product and coordinate axes. Assuming given coordinates, vector product is given by

$$
\begin{aligned}
& |\mathbf{R S} \times \mathbf{S ~ T}|= \\
& =\left\{\left[\left(y_{2}-y_{1}\right)\left(z-z_{2}\right)-\left(z_{2}-z_{1}\right)\left(y-y_{2}\right)\right]^{2}+\right. \\
& +\left[\left(z_{2}-z_{1}\right)\left(x-x_{2}\right)-\left(x_{2}-x_{1}\right)\left(z-z_{2}\right)\right]^{2}+ \\
& \left.+\left[\left(x_{2}-x_{1}\right)\left(y-y_{2}\right)-\left(y_{2}-y_{1}\right)\left(x-x_{2}\right)\right]^{2}\right\}^{\frac{1}{2}} .
\end{aligned}
$$

Cosines of the angle formed by this vector (and, hence, magnetic induction vector) and coordinate axes are:

$$
\left\{\begin{array}{l}
\cos \gamma_{x}=\frac{\left(y_{2}-y_{1}\right)\left(z-z_{2}\right)-\left(z_{2}-z_{1}\right)\left(y-y_{2}\right)}{|\mathbf{R S} \times \mathbf{S T}|}, \\
\cos \gamma_{y}=\frac{\left(z_{2}-z_{1}\right)\left(x-x_{2}\right)-\left(x_{2}-x_{1}\right)\left(z-z_{2}\right)}{|\mathbf{R S} \times \mathbf{S T}|}, \\
\cos \gamma_{z}=\frac{\left(x_{2}-x_{1}\right)\left(y-y_{2}\right)-\left(y_{2}-y_{1}\right)\left(x-x_{2}\right)}{|\mathbf{R S} \times \mathbf{S T}|} .
\end{array}\right.
$$

The components of the magnetic induction vector for the time $\mathrm{t}$ and point $T(x, y, z)$ originating from $k^{\text {th }}$ segment are given by:

$$
\left\{\begin{array}{l}
b_{x, k}(t)=\cos \gamma_{x} b_{k}(t) \\
b_{y, k}(t)=\cos \gamma_{y} b_{k}(t) \\
b_{z, k}(t)=\cos \gamma_{z} b_{k}(t)
\end{array}\right.
$$


Total value of the magnetic induction vector is given by summing contributions from all $n$ segments

$$
b(t)=\sqrt{\left[\sum_{k=1}^{n} b_{x, i}(t)\right]^{2}+\left[\sum_{k=1}^{n} b_{y, i}(t)\right]^{2}+\left[\sum_{k=1}^{n} b_{z, i}(t)\right]^{2}} .
$$

The obtained magnetic induction value is time-dependent, which is the direct consequence of the time-dependent current flowing through the conductors. In order to determine magnetic induction level, one needs to apply the root mean square (RMS) value. Aiming towards the reliable calculation of the RMS, it is necessary to calculate the magnetic induction during the at least one period of the fundamental harmonic $f_{l}$.

\section{Magnetic InduCtion Simulation Results}

Aforementioned algorithm is applied to obtain values of the magnetic induction for 2 types of the overhead power lines. Results are presented graphically. Fig. 2 displays the space position of the conductors in case of cross-section perpendicular to power line route in the sag location, for 400 $\mathrm{kV}$ power line. Same figure depicts the iso-lines for the magnetic induction RMS for currents of $450 \mathrm{~A}, 50 \mathrm{~Hz}$.

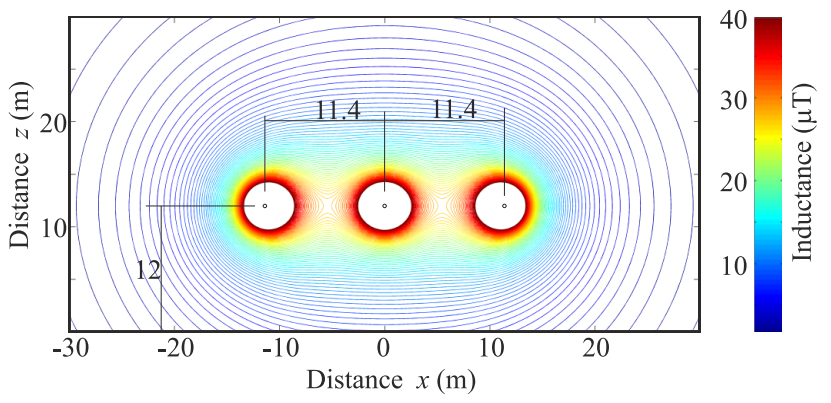

Fig. 2. Space distribution of the $400 \mathrm{kV}$ overhead power line conductors and iso-lines of the magnetic induction RMS for current of $450 \mathrm{~A}, 50 \mathrm{~Hz}$.

The value of the magnetic induction observed on the specific aboveground height depends on distance to conductor sag. Fig. 3 displays magnetic induction value changes along $400 \mathrm{kV}$ power line axis with respect to the magnetic induction under the sag.

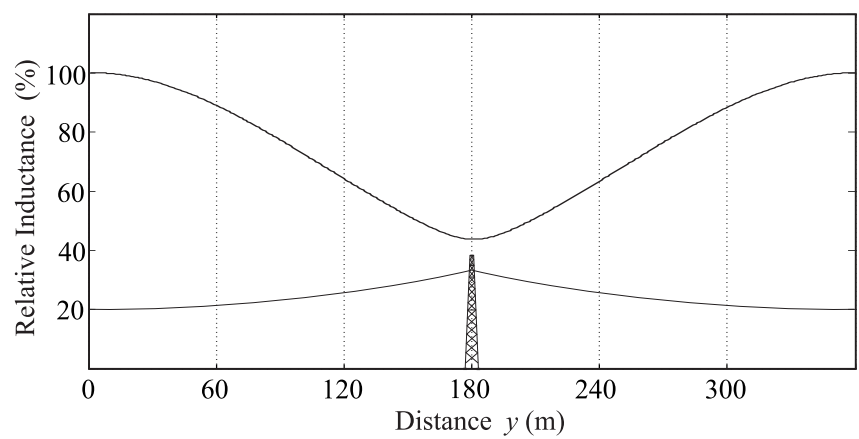

Fig. 3. Magnetic induction value alterations along $400 \mathrm{kV}$ overhead power line axis.

Observation line is fixed on the height of $1 \mathrm{~m}$, its total length is $360 \mathrm{~m}$, the location is fixed between the two sags, while sag height is equal to $12 \mathrm{~m}$. The value of the magnetic induction alters significantly along the power line axis. Magnetic induction determined on the conductor hanging location is $45 \%$ lower compared to the induction measured on the sag location. It is worth of mentioning that simulated value under the sag location is differing from simulated value when influence of the conductor curvature (catenary equation) is neglected for less than $1 \%$. Consequently, this means that in order to determine the magnetic induction maxima one can apply simple model assuming long plane conductors placed on the height of the curved conductor sag.

\section{COMPARISON OF THE CALCUlATED AND MEASURED RESULTS}

Proposed algorithm is verified by comparison with the measurements executed on the 3-phase overhead power lines, $110 \mathrm{kV}$ type "fir" and $400 \mathrm{kV}$ type "Y" passing over Industrial zone Dugopolje. As previously stated, currents measured on the $400 \mathrm{kV}$ "Y" overhead power line are higher resulting is higher magnetic flux density values, that is why we omit $110 \mathrm{kV}$ "fir" power line from further discussion.

In case the accurate measurements are desired, the magnetic induction in the outdoor conditions is usually measured via so called field-analyzers. Field analyzers are devices enabling field of interest spectral analysis [14]. Basic problem with magnetic field measurements are probes, more precisely its response dependence to the position with respect to the field source [15]. This problem is circumvented by using the probe based on tree mutually perpendicular coils. The benefit of the aforementioned arrangement is that the sensor provides the same magnetic field readout, regardless the sensor-field orientation. However, it should be pointed out that even small deviations of the coils within the probe (with respect to their ideal positions) induce errors of order of $2 \%$ or more [15].

Field analyzer used in this research is equipped with 3 independent mutually perpendicular coils that measure all 3 space components mutually. Regardless of the instrument orientation, display value is equal to total magnetic induction RMS.

Man located in the vicinity of the ELF radiation source is exposed to component of the magnetic induction perpendicular to the ground [14]. Fig. 4 reports measured values of the magnetic induction RMS on location $1 \mathrm{~m}$ above ground, meaning $11 \mathrm{~m}$ under lowest conductor height in case of $400 \mathrm{kV}$ with the current of $140 \mathrm{~A}$. Same figure reports RMS values of the simulated induction in case of the total value and $\mathrm{x}$ and $\mathrm{z}$ axis component separated. $110 \mathrm{kV}$ case is omitted from the analysis, but due to reasons of the distinctness, we would like to point out that $110 \mathrm{kV}$ ("fir") results are consistent and in accordance to $400 \mathrm{kV}$ equivalents.

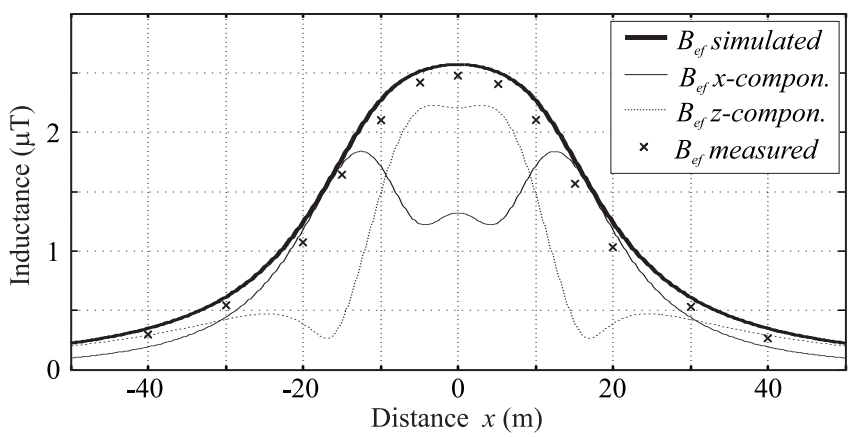

Fig. 4. Measured and simulated magnetic induction RMS values for the $400 \mathrm{kV}$ overhead power line. 
The satisfactorily agreement between measured and simulated values is observable. Small differences could be attributed to the complexity of the ground configuration, lack of the possibility to obtain the exact power line geometry data and non-simultaneous measurements (current oscillations within the 15 min measuring intervals).

According to the technical regulations regarding the safety distance from the biased elements [11], the safety corridors along the overhead power lines are defined. Assuming the fact that the general population should not sojourn in the immediate vicinity of the power line, the measurements and the estimation of the magnetic induction should be done on the border of the safety corridor. The width of the safety corridor is $50 \mathrm{~m}$ and $60 \mathrm{~m}$ for the $110 \mathrm{kV}$ and $400 \mathrm{kV}$ overhead power line, respectively. Fig. 5 reports magnetic induction at the very edge of the safety corridor in case of $400 \mathrm{kV}$ flowed by $180 \mathrm{~A}$ and $320 \mathrm{~A}$ of current.

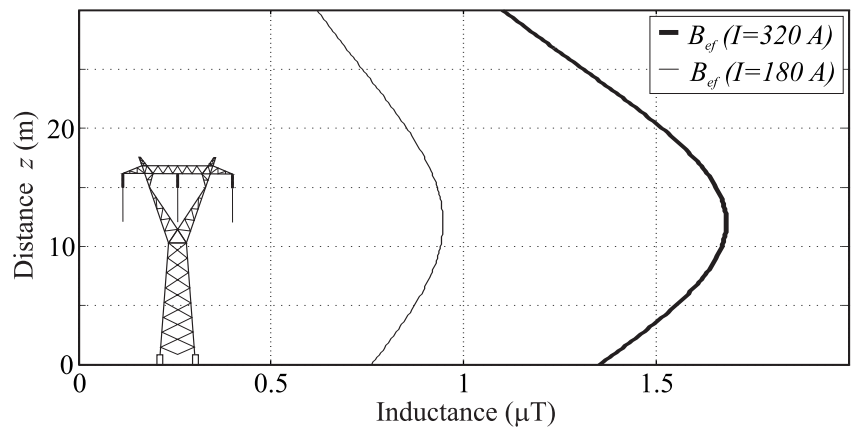

Fig. 5. Magnetic induction at the border of safety corridor for $400 \mathrm{kV}$ overhead power line.

\section{COMPARISON OF THE OBTAINED MAGNETIC INDUCTION VALUES AND THE REFERENT VALUES}

The magnitude of the magnetic flux density is determined by power/current transmitted over the power line. The most critical parameter to determine magnetic flux density from the power line model is the current flowing through the power line. The power line current is stochastic value; hence, refferent power line current is determined statistically from the long-term measurements. Based on the available data regarding the average daily currents within 4 months period, we obtained the most commonly observed current magnitude, referent for the magnetic field estimation from the power line model. Furthermore, measurements performed in order to evaluate accuracy of the model have been performed at current exactly equal to this statistically obtained (most frequently present) magnitude. The maximal calculated magnetic induction values, in case of both types of power lines, are compared to referent values in [11]. According to [16], critical exposure period is determined by human body ability to compensate continuous overexposure without the harmful consequences. If the field strength increases, human body can activate defense mechanisms (eg. sweating), assuming that exposed interval is short enough. From medical research [17], it is known that human body can ensure proper thermoregulation mechanisms only if the exposed period for magnetic field inductions higher than limits stated in INCNIRP recommendation [16] is shorter than $6 \mathrm{~min}$. The Croatian regulations define more strict limits, meaning, the magnetic field inductions lower than stated in [11] are not proved to be dangerous for the human beings no matter the duration of the exposure period. Due to sake of clarity, we point out that the ICNIRP limiting value of magnetic field is potentially dangerous if the exposure period is longer than $6 \mathrm{~min}$, double limiting value is dangerous after $3 \mathrm{~min}$, etc.

The magnetic induction maxima with value of $2.57 \mu \mathrm{T}$ is significantly lower than the limits of $100 \mu \mathrm{T}$ for the workers and $40 \mu \mathrm{T}$ for the general population given by the Regulation of the Croatian Ministry of Health [11] and, hence, according to [17] does not present the potential health treat.

\section{CONCLUSIONS}

We reported the simple model for estimation of the human exposure to ELF magnetic fields produced by overhead power lines. The results obtained from the calculations based on the simplified model are compared to the results of the measurements, resulting in high agreement between measurements and proposed model. Furthermore, it was found that the magnetic field induction under the sag in the case of the catenary shaped conductor model differs from straight conductor model for less than $1 \%$. Finally, in order to determine possible health risks, the obtained results for magnetic induction are compared to the limits given by Croatian laws regarding EM field protection and are found to be significantly lower than aforementioned limits. Future work will extend the model by encompassing possible ELF magnetic fields influence on power line communications in the frame of the smart-grid-related applications.

\section{REFERENCES}

[1] J. D. Jackson, Classical Electrodynamics. John Wiley and Sons, 1962.

[2] Z. Psenakova, J. Hudecova, "Influence of Electromagnetic Fields by Electronic Implants in Medicine", Elektronika ir Elektrotechnika (Electronics and Electrical Engineering), no. 7, pp. 37-40, 2009.

[3] R. W. P. King, "Fields and Currents in the Organs of the Human Body When exposed to Power Lines and VLF Transmitters", IEEE trans. vol. 45, no. 4, pp. 520-530, 1998.

[4] "Electric and Magnetic Fields Associated with the Use of Electric Power", National Institute of Environmental Health Sciences, 2002

[5] International agency for research on cancer, Press release, no. 208, 2011.

[6] S. J. Genius, "Fielding of the current idea: Exploring the public health impact of EM radiation", Public health, pp. 1-12, 2007.

[7] R. G. Olsen, P. S. Wong, "Characteristics of Low Frequency Electric and Magnetic Fields in the Vicinity of the Electric Power Lines", IEE Trans on Power delivery, vol. 7, no. 4, pp.2046-2053, Oct. 1992.

[8] H. Haase, J. Nitsch, "Full-Wave Transmission Line Theory fo the Analysis of the Three-Dimentional Wire-Like Structures", in Proc. of $14^{\text {th }}$ International Symposium on EM, Zurich, 2000, pp. 235-240.

[9] A. Cheldavi, "Radiation from the Coupled Multiconductor Transmission Lines", IEEE Proc., vol. 150, no. 1, pp. 15-22, 2000.

[10] P. Pahrami, R. Mittra, "Wire Antennas over a Lossy Half-Space", IEE trans, vol. 28, no. 3, 1980.

[11] Regulation on EM field protection, Official Gazette 204, Dec. 2003.

[12] T. Bosanac, Theoretical basics of the Electrical engineering, Part I, TK Zagreb, 1970.

[13] W. H. Beyer, CRC Standard Mathematical Tables, 28th ed., Boca Raton, FL: CRC Press, 1987, p. 214.

[14] S. Šesnić, "Electromagnetic Modeling of the Human Body Exposed to Extremely Low Frequencies", M.S. thesis, WIT, Wales, 2004.

[15] H.-J. Foerster, The Current State of the Field Measurement Technology. Wandel \& Goltermann GmbH \& Co, Eningen, 2013

[16] D. Poljak, Human Exposure to Non-Ionization Radiation, Kigen, Zagreb, 2006.

[17] "Possible effects of Electromagnetic Fields (EMF) on Human Health", Scientific Committee on Emerging and Newly Identified Health Risks (SCENIHR), European Commission, 2007. 